Supporting Information

\title{
Layered Lithium-Rich Oxide Nanoparticles Doped with Spinel Phase: Acidic Sucrose-Assistant Synthesis and Excellent Performance as Cathode of Lithium Ion Battery
}

Min Chen ${ }^{\mathrm{a}}$, Dongrui Chen ${ }^{\mathrm{a}}$, Youhao Liao ${ }^{\mathrm{a}, \mathrm{b}}$, Xiaoxin Zhong ${ }^{\mathrm{a}}$, Weishan Li ${ }^{\mathrm{a}, b^{*}}$, Yuegang Zhang $^{\mathrm{a}}$

a. School of Chemistry and Environment, South China Normal University, Guangzhou 510631, China

b. Engineering Research Center of MTEES (Ministry of Education), Research Center of BMET (Guangdong Province), Engineering Lab. of OFMHEB (Guangdong Province), Key Lab. of ETESPG (GHEI), and Innovative Platform for ITBMD (Guangzhou Municipality), South China Normal University, Guangzhou 510006, China

\section{Corresponding Author}

*liwsh@scnu.edu.cn 


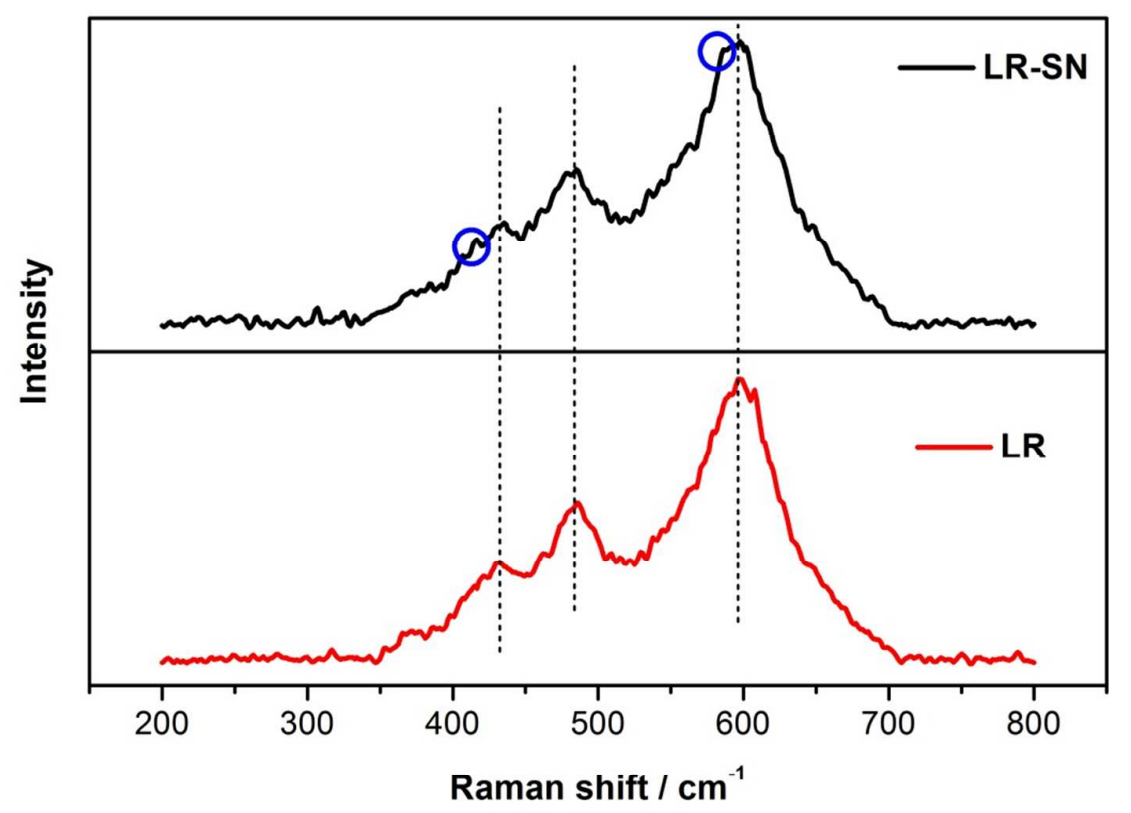

Fig. S1. Raman spectra of LR-SN and LR. 

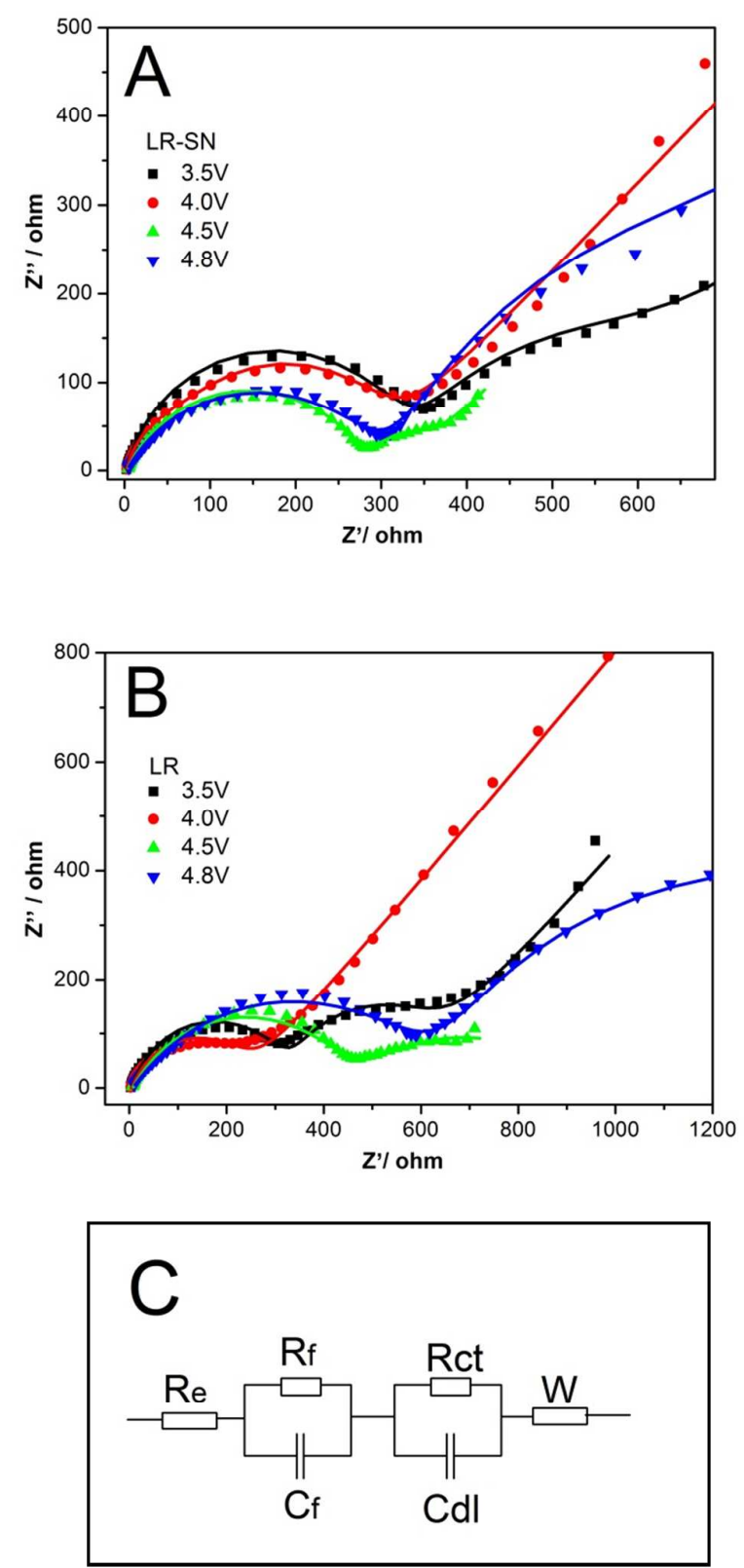

Fig. S2. Electrochemical impedance spectra of LR-SN (A) and LR(B) in the initial cycle at different charge states and equivalent circuit $(C)$ for fitting. 

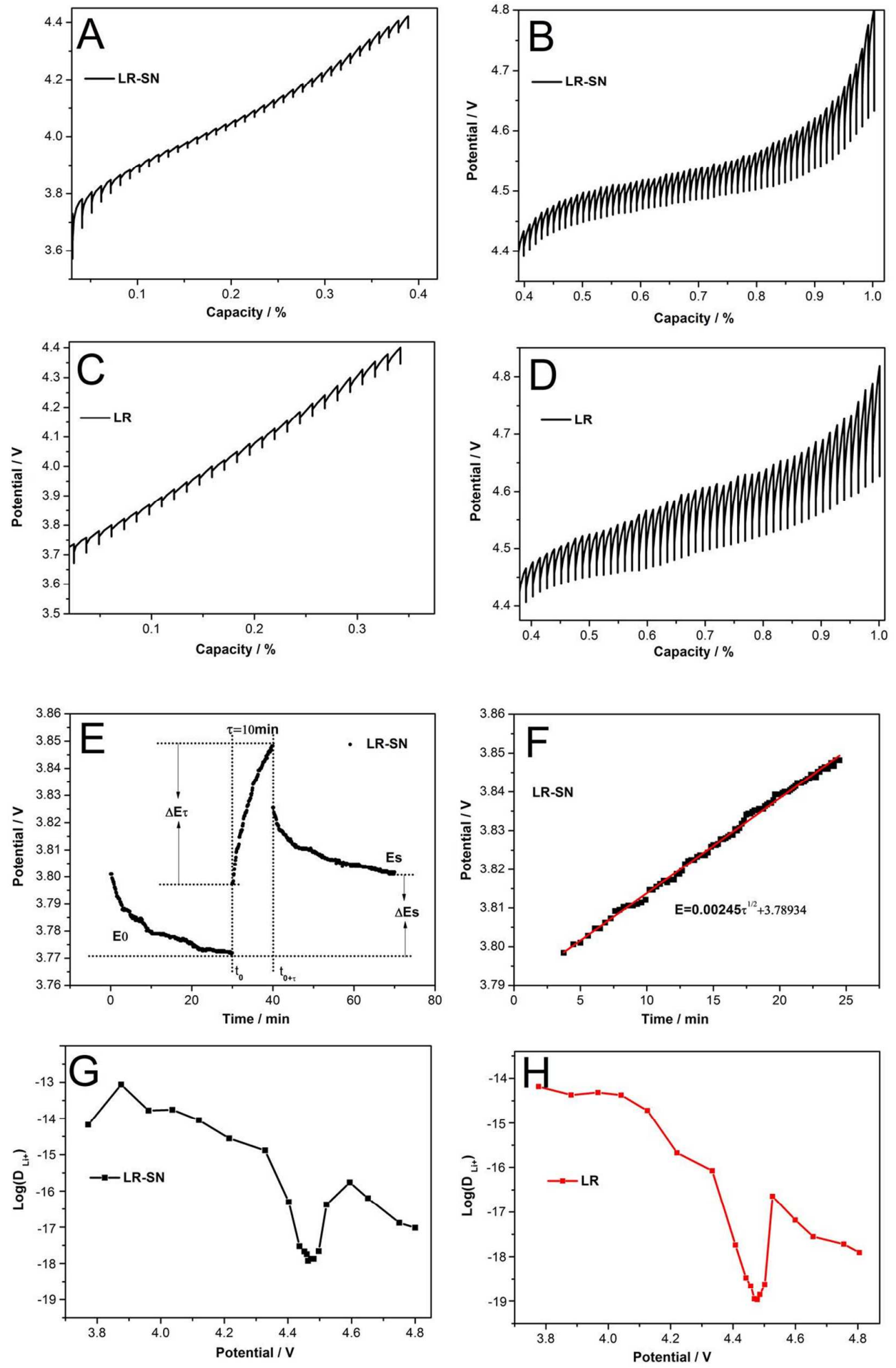

Fig. S3. GITT curves of LR-SN (A and B) and LR (C and D) for the initial cycle between $2.0 \mathrm{~V}$ and $4.8 \mathrm{~V}$ (current flux: $25 \mathrm{~mA} \mathrm{~g}^{-1}$, time interval: $30 \mathrm{~min}$ ), $\mathrm{t}$ vs.E profile of LR-SN for a selected single GITT titration (E), linear behavior of E vs. $s^{1 / 2}$ $(\mathrm{F})$, and diffusion coefficients of LR-SN $(\mathrm{G})$ and $\mathrm{LR}(\mathrm{H})$ at different charge states. 


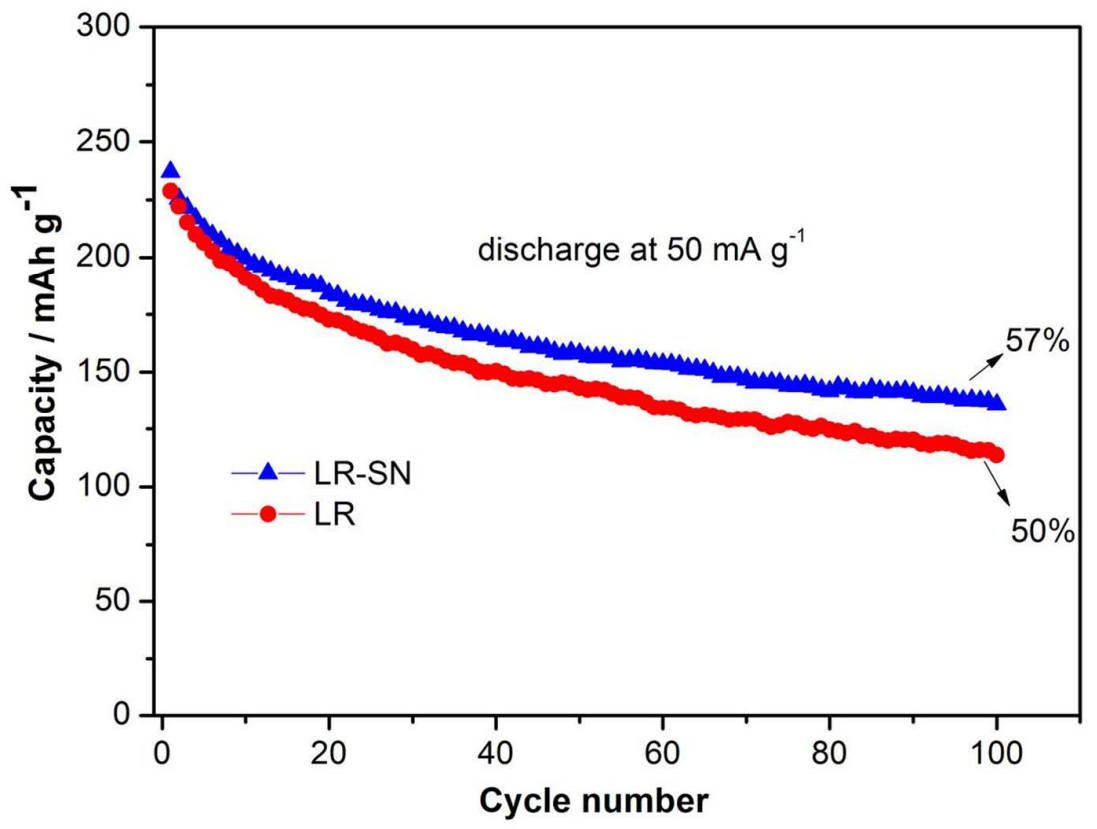

Fig. S4. Cyclic stability of LR-SN and LR between 2.0-4.8 V at a current density of $50 \mathrm{~mA} \mathrm{~g}^{-1}$ 
Table S1. Fitting parameters for electrochemical impedance spectra of two samples at different potentials.

\begin{tabular}{lccccc}
\hline Potential(V) & Samples & $\mathrm{R}_{\mathrm{e}}(\Omega)$ & $\mathrm{R}_{\mathrm{f}}(\Omega)$ & $\mathrm{R}_{\mathrm{ct}}(\Omega)$ & $\mathrm{R}_{\text {total }}(\Omega)$ \\
\hline \multirow{2}{*}{$3.5 \mathrm{~V}$} & LR & 1.9 & 323 & 240 & 564.9 \\
& LR-SN & 1.6 & 320 & 230 & 551.6 \\
$4.0 \mathrm{~V}$ & LR & 2 & 220 & 110 & 332 \\
& LR-SN & 1.9 & 215 & 60 & 276.9 \\
$4.5 \mathrm{~V}$ & LR & 4.0 & 438 & 500 & 942 \\
& LR-SN & 3.0 & 280 & 150 & 433 \\
$4.8 \mathrm{~V}$ & LR & 4.2 & 625 & 653 & 629.2 \\
& LR-SN & 3.0 & 280 & 225 & 508 \\
\hline
\end{tabular}

